\title{
A Direct Approach to Organic/Inorganic Semiconductor Hybrid Particles via Functionalized Polyfluorene Ligands
}

\author{
Tjaard de Roo, Johannes Haase, Janine Keller, Christopher Hinz, Marius Schmid, \\ Denis V. Seletskiy, Helmut Cölfen, Alfred Leitenstorfer, and Stefan Mecking**
}

\begin{abstract}
Controlled Suzuki-Miyaura coupling polymerization of $7^{\prime}$-bromo-9', $9^{\prime}$-dioctylfluoren-2'-yl-4,4,5,5-tetramethyl-[1,3,2]dioxaborolane initiated by bromo(4tert-butoxycarbonylamino-phenyl)(tri-tert-butylphosphine)palladium (1) or bromo(4-diethoxyphosphoryl-phenyl)(tri-tert-butylphosphine)palladium (2) yields functionalized polyfluorenes $\left(M_{n}=4 \times 10^{3} \mathrm{~g} \mathrm{~mol}^{-1}, M_{w} / M_{n}<1.2\right)$ with a single amine or phosphonic acid, respectively, end-group. High temperature synthesis of cadmium selenide quantum dots with these functionalized polyfluorenes as stabilizing ligands yields hybrid particles consisting of good quality (e.g. emission full width at half maximum of $30 \mathrm{~nm}$; size distribution $\sigma<10 \%)$ inorganic nanocrystals with polyfluorene attached to the surface, as corroborated by transmission electron microscopy analysis and analytical ultracentrifugation. Sedimentation studies on particle dispersions show that a substantial portion (ca. half) of the phosphonic acid terminated polyfluorene ligands is bound to the inorganic nanocrystals, versus ca. $5 \%$ for the aminofunctionalized polyfluorene ligands. Single particle micro-photoluminescence spectroscopy shows an efficient and complete energy transfer from the polyfluorene layer to the inorganic quantum dot.
\end{abstract}

\section{Introduction}

Hybrid nanoparticles comprised of inorganic semiconductor quantum dots (QDs) and organic semiconductor conjugated polymers have recently gained considerable interest. They allow for processing to highly disperse organic/inorganic composite bulk materials, which are difficult to access otherwise. Due to a possible charge transport and separation at the organic/inorganic semiconductor interface, these composites are interesting

T. de Roo, S. Mecking

Chair of Chemical Materials Science

University of Konstanz

D-78457, Germany

E-mail: Stefan.Mecking@uni-konstanz.de

M. Schmid, H. Cölfen

Chair of Physical Chemistry

Department of Chemistry

University of Konstanz

D-78457, Germany

J. Haase, J. Keller, C. Hinz, D. V. Seletskiy, A. Leitenstorfer Department of Physics and Center for Applied Photonics

University of Konstanz

D-78457, Germany candidates for photonic applications (e.g. light emitting diodes, ${ }^{[1-3]}$ or photovoltaics $^{[4-7]}$ ) or flexible electronics. ${ }^{[8]}$ Multiple QDs in a conjugated polymer nanoparticle have been suggested as bright redemitting probes for biological imaging, ${ }^{[9]}$ whereas polymer particles containing single QDs are of particular interest for quantum photonic applications. The latter benefit from an increase in the long-term stability of the embedded QD. ${ }^{[10]}$ In particular, our motivation is to synthesize nanoparticles from a combination of CdSe QDs with polyfluorene $(\mathrm{PF})^{[11]}$ as the conjugated polymer, with the long term aim of a controlled generation of singlycharged colloidal QDs. This would be an ideal system for quantum optical experiments where the individual addressability and manipulability ${ }^{[12]}$ of singly charged pure two-level systems is needed. ${ }^{[13-16]}$

Currently, the synthesis of hybrid particles remains a challenging task and most approaches are based on ligand exchange procedures. That is, organic ligands, employed during the quantum dot synthesis, are subsequently replaced by the actually desired functional ligands. ${ }^{[17,18]}$ For example the synthesis of polyfluorene-functionalized $\mathrm{CdSe} / \mathrm{ZnS}$ QDs by replacing pyridine ligands with polyfluorene derivatives with amino-functionalized side chains has been reported. ${ }^{[19]}$ However, the ligand exchange is accompanied by disadvantages such as incomplete replacement of the original ligands, surface oxidation and a reduction in quantum yield. ${ }^{[20]}$ In order to avoid the need for a ligand exchange, one can use functionalized ligands during QD synthesis combined with a subsequent "grafting from" or a "grafting onto"[21] approach by polymerizing from- or attaching a pre-synthesized polymer to the QD surface. An example for the "grafting from" strategy was reported by Emrick et al. where a bromine functionalized ligand was used during QD synthesis. Subsequent oligomerization yielded CdSe QDs decorated with oligo(p-phenylen-vinylen) tri- and tetramers as ligands. ${ }^{20]}$

An unresolved issue, however, in all these studies is whether and to what extent the conjugated polymer is actually attached to the inorganic portion. This is due to the shortage of appropriate analytics which directly address the particles in liquid dispersion. For example, conventional transmission electron microscopy as a well-established technique involves sample preparation by drying on a grid. Solutes which are not attached 
to the inorganic particles in the original dispersion nonetheless will often accumulate on the particles during sample preparation by capillary forces.

Here we report a novel approach to produce hybrid particles directly by the high-temperature synthesis of inorganic semiconductor quantum dots in the presence of conjugated polymer ligands. Monofunctionalized polyfluorene required for this purpose is generated by living polymerization from amino and phosphonate functionalized initiators. Binding of these tailored polyfluorenes to the inorganic nanocrystals is elucidated by analytical ultracentrifugation.

\section{Results and Discussion}

Controlled Suzuki-Miyaura coupling polymerization was used for the synthesis of functionalized polyfluorenes, as first reported by Yokozawa et al. in 2007.[22] For this living polymerization technique, a T-shaped $\mathrm{Pd}(\mathrm{II})$ complex, carrying a phosphine, a bromine, and a phenyl ligand, is used as an initiator. After polymerization, the phenyl ligand ends up at every polymer chain-end. ${ }^{[22-25]}$ The power of this method for generating functional end-groups was recently demonstrated by us by using T-shaped Pd(II) complexes as initiators carrying protected hydroxyl-functions at the phenyl ligand, leading to hydroxyl-functionalized polyfluorenes after a consecutive deprotection step. ${ }^{[26]}$ However, the hydroxyl functionality reported in this prior work is unsuitable for the interaction with the surface of inorganic CdSe semiconductor quantum dots, as required for the approach pursued here. For this reason, T-shaped Pd(II) complexes featuring amine and phosphonic acid functional groups in a protected form were synthesized and used as initiators for the living Suzuki-Miyaura coupling polymerization (Scheme 1).

To avoid side reactions during initiator synthesis as well as during the polymerization procedure, the amine functionality was protected with the tert-butyloxycarbonyl (BOC) protecting group and the phosphonic acid functionality was introduced as a diethylester. These protecting groups were chosen because of their stability under the basic polymerization conditions and their ability for a straightforward removal by heating. The initiator complexes were synthesized by oxidative addition of an excess ( 3 equiv.) of the respective aryl bromides to $\left[\mathrm{Pd}\left(\mathrm{P}^{t} \mathrm{Bu}_{3}\right)_{2}\right]$ in butanone at $75{ }^{\circ} \mathrm{C}$. Both complexes were isolated and purified by precipitation in pentane after removal of butanone with yields of $71 \%$ and $46 \%$ for initiator 1 and 2, respectively and characterized by ${ }^{1} \mathrm{H}$-NMR- and ${ }^{31} \mathrm{P}-\mathrm{NMR}$ spectra, elemental analysis and ESI-MS spectra (cf. Supporting Information, SI). Both complexes effectively initiate the polymerization of 7'-bromo-9', $9^{\prime}$-dioctyl-fluoren-2'-yl-4,4,5,5-tetramethyl-[1,3,2] dioxaborolane (3), leading to the respective BOC-protected amino-, or phosphonic acid diester-functionalized polyfluorene with narrow molecular weight distribution $\left(\mathrm{M}_{\mathrm{w}} / \mathrm{M}_{\mathrm{n}}<1.2\right)$ and molecular weight $\left(\mathrm{M}_{\mathrm{n}}\right)$ of $4 \times 10^{3} \mathrm{~g} \mathrm{~mol}^{-1}$ respectively, underlining the controlled character of the Suzuki-Miyaura coupling polymerization. MALDI-TOF mass- and ${ }^{1} \mathrm{H}-\mathrm{NMR}$ spectra clearly evidence that every polymer chain is terminated with the respective aryl moiety from the applied initiators, indicating an effective initiation of the polymerization by complexes 1 and 2 (SI).

According to MALDI-TOF mass spectra, the terminal ends of the polyfluorene chains are substituted by hydrogen with only small amount of polyfluorene chains with bromine endgroups $(<10 \%)(\mathrm{SI})$. Quenching with concentrated $\mathrm{HCl}$ during work-up of the polymerization mixture already resulted in a partial deprotection $(\approx 20 \%)$ by cleavage of the BOC group in case of the polymerization initiated by complex 1 (SI). For complete removal of the BOC protecting group, the polyfluorene was heated under reduced pressure to $190{ }^{\circ} \mathrm{C}$, resulting in a loss of isobutene and carbon dioxide and the formation of the amine functionality. Likewise, phosphonic acid terminated polyfluorene was obtained by heating to $240{ }^{\circ} \mathrm{C}$ under reduced pressure resulting in a loss of ethylene (Scheme 1). Complete deprotection in both cases was confirmed by MALDI-TOF mass (Figure 1) and ${ }^{1} \mathrm{H}$-NMR spectra (SI). ${ }^{1} \mathrm{H}$-NMR spectra showed the functionalized fluorenes to be stable at temperatures of $240{ }^{\circ} \mathrm{C}$ over 10 minutes, as no changes could be observed.

Cadmium selenide nanocrystals were prepared by a hot injection $^{[27]}$ approach at a temperature of $240{ }^{\circ} \mathrm{C}$. From intensive studies of such syntheses aiming at the control of optical quality via nanocrystal sizes and size distribution, protocols employing oleyl amine and alkylphosphonic acids as stabilizing ligands have been found empirically. ${ }^{[10,28,29]}$ For the synthesis of hybrid particles, the functionalized polyfluorene ligands were directly added to the synthesis of the CdSe QDs, partially replacing oleyl amine or dodecylphosphonic acid. However, oleyl amine could not be replaced by the amino-functionalized polyfluorene stoichiometrically, as oleyl amine is used in a large excess and a corresponding molar amount of polyfluorene is insoluble in the reaction mixture. Therefore, QDs were synthesized using oleyl

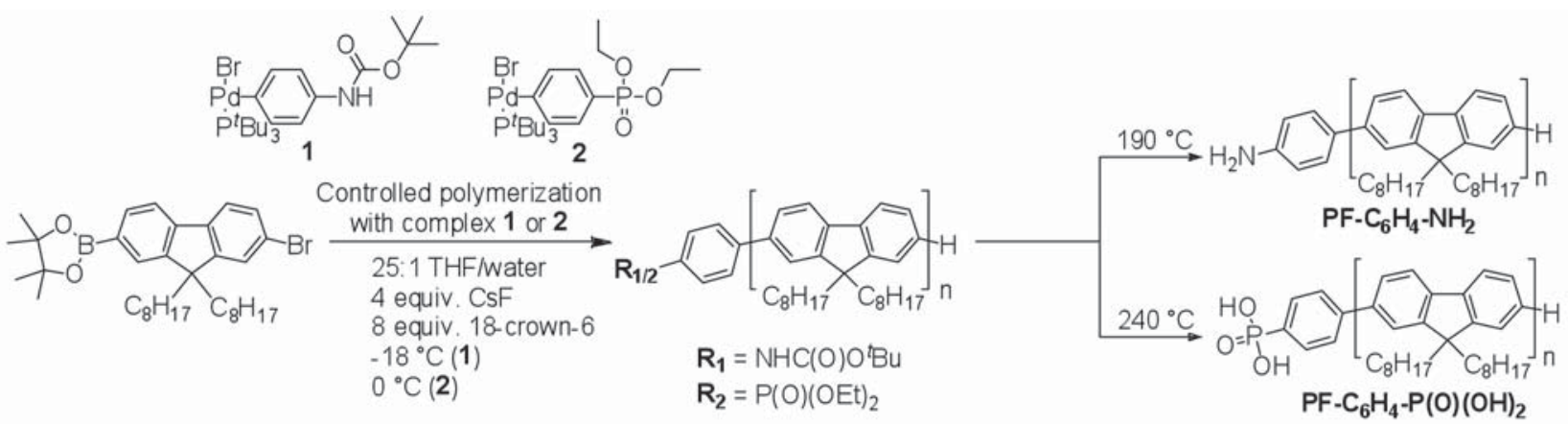

Scheme 1. Complexes 1 and 2 with protected functional groups and synthesis of functionalized polyfluorenes $\mathbf{P F}-\mathrm{C}_{6} \mathrm{H}_{4}-\mathrm{NH} \mathrm{H}_{2}$ and $\mathbf{P F}-\mathrm{C}_{4} \mathrm{H}_{4}-\mathrm{P}(\mathrm{O})(\mathrm{OH})_{4}$. 


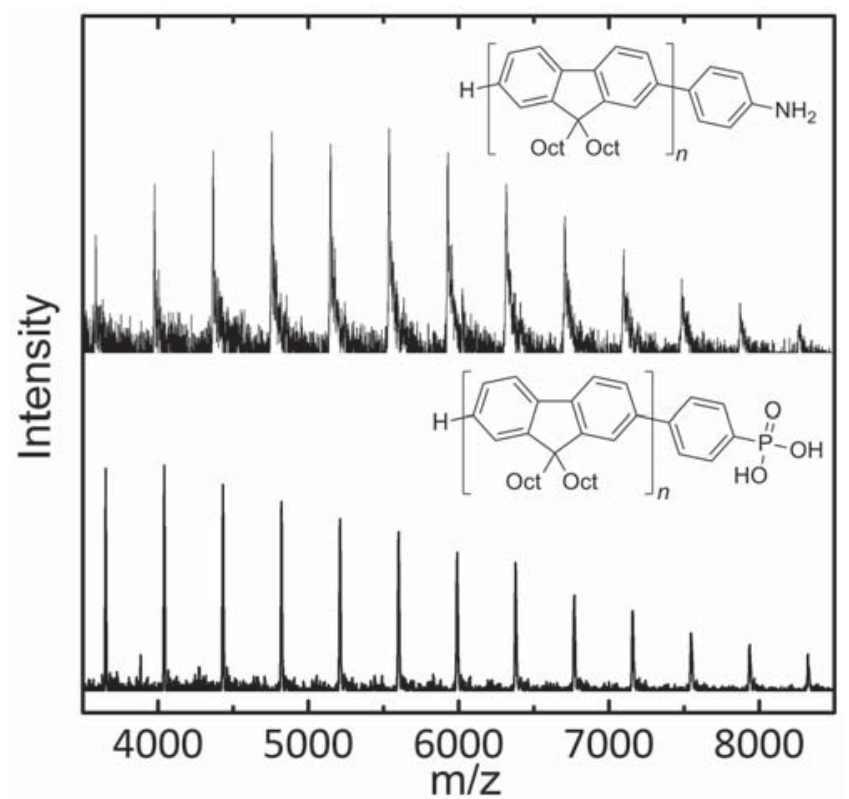

Figure 1. MALDI-TOF mass spectra of functionalized polyfluorenes (with $n$ ranging from 9 to 21 ) after deprotection: $\mathrm{PF}-\mathrm{C}_{6} \mathrm{H}_{4}-\mathrm{NH}_{2}$ (top) and PF- $\mathrm{C}_{6} \mathrm{H}_{4}-\mathrm{P}(\mathrm{O})(\mathrm{OH})_{2}$ (bottom).

amine, dodecylphosphonic acid and additional amino-functionalized polyfluorene (0.01 equiv. with respect to oleyl amine) as ligands. The resulting hybrid nanoparticles were studied by transmission electron microscopy (TEM), yielding size distributions of the nanocrystals (Figure 2). The QDs synthesized with $\mathrm{PF}-\mathrm{C}_{6} \mathrm{H}_{4}-\mathrm{NH}_{2}$ as an additional ligand together with oleyl amine and dodecylphosphonic acid $\left(\mathrm{QDs}\left(\mathrm{H}_{2} \mathrm{~N}-\mathrm{C}_{6} \mathrm{H}_{4}-\mathrm{PF}\right)\right)$, show a mean diameter of $4.6 \mathrm{~nm}$ with a standard deviation of $\sigma=$ $0.40 \mathrm{~nm}$ (Figure 2a, $\mathbf{Q D s}\left(\mathrm{H}_{2} \mathbf{N}-\mathrm{C}_{6} \mathrm{H}_{4}-\mathrm{PF}\right)$ ). In contrast, the reference QDs synthesized in the presence of only oleyl amine and dodecylphosphonic acid, without polyfluorene as an additional ligand ( $Q \mathbf{Q s}_{\text {ref }}$, Figure $2 \mathrm{~b}$ ), exhibit a mean diameter of $4.3 \mathrm{~nm}$ with a slightly broader size distribution $(\sigma=0.42 \mathrm{~nm})$. This analysis is independently corroborated by optical emission

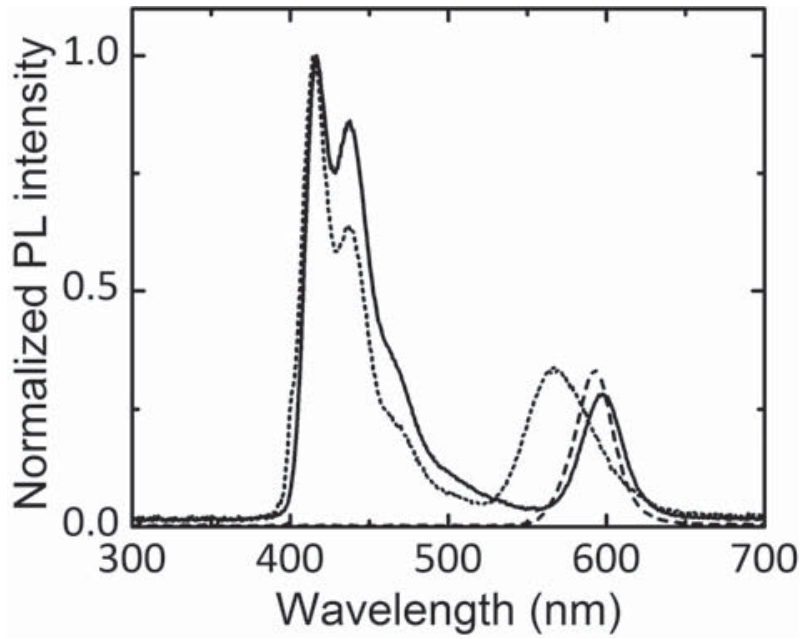

Figure 3. Photoluminescence spectra of QDs prepared using the following ligands: oleyl amine, dodecylphosphonic acid and amino-functionalized polyfluorene (QDs $\left.\left(\mathrm{H}_{2} \mathbf{N}-\mathbf{C}_{6} \mathrm{H}_{4}-\mathrm{PF}\right)\right)$, solid curve, $\mathrm{FWHM}=$ $30 \mathrm{~nm}$ ); oleyl amine and phosphonic acid functionalized polyfluorene (QDs((HO) $\left.{ }_{2}(\mathrm{O}) \mathrm{P}-\mathrm{C}_{6} \mathrm{H}_{4}-\mathrm{PF}\right)$ ), dotted curve, FWHM = $48 \mathrm{~nm}$ ); oleyl amine and dodecylphosphonic acid $\left(\right.$ QDs $_{\text {ref }}$, dashed curve, FWHM = $31 \mathrm{~nm}$ ).

spectra of the corresponding QD ensembles (Figure 3). A comparison of the photoluminescence line shapes shows that QDs $\left(\mathrm{H}_{2} \mathbf{N}-\mathrm{C}_{6} \mathrm{H}_{4}-\mathrm{PF}\right)$ emission is slightly narrower (solid curve, FWHM $=30 \mathrm{~nm}$ ) and occurs at a higher wavelength, as compared to the QDs $\mathbf{r e f}_{\text {re }}$ emission (dashed curve, FWHM = $31 \mathrm{~nm}$ ). Narrow optical line width indicates correspondingly narrow size distribution, while red/blue shift of the emission corresponds to an increase/decrease of the average size of the nanocrystals. Thus, spectra of the optical emission from the nanocrystals are in full qualitative agreement with the above TEM analysis.

In the above studies, the polyfluorene ligand will compete with the other ligands present, oleyl amine and dodecylphosphonic acid, for coordination sites at the QD surface. It must be assumed that the steric demand of the polyfluorene hinders its binding vs. the other ligands. Additionally, it is
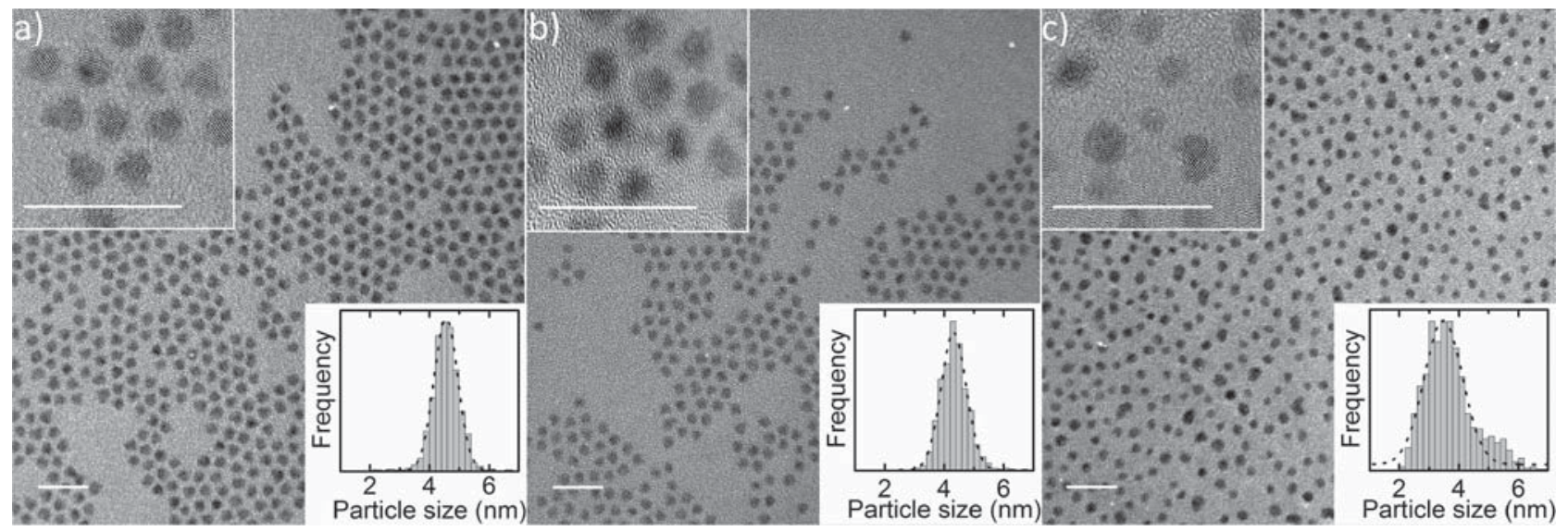

Figure 2. Transmission electron micrographs and particle size distribution histograms (number of particles counted > 600) with Gaussian fits (dotted curves) of quantum dots synthesized using the following ligands: a) oleyl amine, dodecylphosphonic acid and amino-functionalized polyfluorene (QDs $\left(\mathrm{H}_{2} \mathbf{N}-\mathbf{C}_{6} \mathrm{H}_{4}-\mathbf{P F}\right)$ ); b) oleyl amine and dodecylphosphonic acid (QDs ref $_{\text {) }}$ c) oleyl amine and phosphonic acid functionalized polyfluorene $\left(\mathrm{QDs}\left((\mathrm{HO})_{2}(\mathrm{O}) \mathrm{P}-\mathrm{C}_{6} \mathrm{H}_{4}-\mathrm{PF}\right)\right)$; the TEM scale bar corresponds to $20 \mathrm{~nm}$. 
presumed that phosphonic acids can bind covalently to the surface of CdSe QDs, whereas amines bind via a weaker coordinative interaction. ${ }^{[30-32]}$ From these considerations, we anticipated that the amount of bound polyfluorene should be increased by employing the stronger binding phosphonic acid and by reducing the amount of competing ligands. Thus, the dodecylphosphonic acid was replaced by $\mathbf{P F}-\mathrm{C}_{6} \mathbf{H}_{4}$ $\mathbf{P}(\mathrm{O})(\mathrm{OH})_{2}$ (0.01 equiv. with respect to the original amount of dodecylphosphonic acid) in order to favor the probability of binding of the functionalized polyfluorene to the QD surface. Again, dodecylphosphonic acid could not be replaced stoichiometrically by the respective functionalized polyfluorene ligand due to solubility issues in the reaction mixture. TEM images of QDs synthesized with oleyl amine and phosphonic acid functionalized polyfluorene used as ligands $\left(\mathrm{QDs}\left((\mathrm{HO})_{2}(\mathrm{O}) \mathrm{P}-\mathrm{C}_{6} \mathrm{H}_{4}-\mathrm{PF}\right)\right)$ indicate a somewhat broader distribution in size and shape and a smaller mean diameter (Figure 2, TEM image c); mean diameter: $3.7 \mathrm{~nm}, \sigma=$ $0.65 \mathrm{~nm})$, as compared to QDs synthesized with oleyl amine and dodecylphosphonic acid used as ligands $\left(\mathbf{Q D s}_{\text {ref }}\right.$, Figure 2, TEM image b); mean diameter: $4.3 \mathrm{~nm}, \sigma=0.42 \mathrm{~nm}$ ). Again, these observations are corroborated by optical spectroscopy, where the broader size distribution is confirmed by an increase of the fluorescence emission band width to $48 \mathrm{~nm}$ FWHM, while the smaller mean diameter is confirmed by the blue shift of the emission wavelength by $25 \mathrm{~nm}$ with respect to the $\mathbf{Q D s _ { \text { ref } }}$ (Figure 3, dotted curve).

Note that due to the absorption of polyfluorene at the excitation wavelength of the QDs and possible QD absorption of polyfluorene fluorescence, it is not possible to quantify quantum yields of the hybrid particles from these experiments. However, the fluorescence spectra demonstrate that the synthesis of CdSe QDs with functionalized polyfluorenes used as ligands leads to fluorescent QDs (Figure 3, solid and dotted curve), thus indicating reasonably high quantum efficiency of the emitters.

To elucidate whether the conjugated polymer and the QDs are indeed bound to each other as hybrid particles, or form a simple physical mixture with non-bound polyfluorenes, the nanoparticle dispersions were subjected to analytical ultracentrifugation using a specialized multi-wavelength detector which is able to detect UV/Vis spectra for all species which are separated in the centrifugal field (MWL-AUC). ${ }^{[33,34]}$ Due to the large difference in mass and density, particles containing QDs will sediment in the gravitational field at a lower rotational speed than any non-bound polyfluorene chains. The different optical absorption spectra of polyfluorene and QDs (SI) allow for tracking of the sedimentation dynamics of both species simultaneously by time-resolved multi-wavelength absorption measurements. Spectral analysis was performed at wavelengths of $380 \mathrm{~nm}$ and $290 \mathrm{~nm}$. At $290 \mathrm{~nm}$, the QD absorption is considerably higher as compared to absorption in polyfluorene, thus contribution of the latter can be ignored at this wavelength (Figure S10). At the same time, contribution of the QDs to the absorption at $380 \mathrm{~nm}$ is not negligible, but can be subtracted employing Lambert-Beer's law. Absorption spectra (Figure 4) of these hybrid particle dispersions are most instructive at three different points in time: before (scan 1; a), during (scan 40; b) and after centrifugation (scan 100; c). Initially, a homogeneous distribution is observed throughout

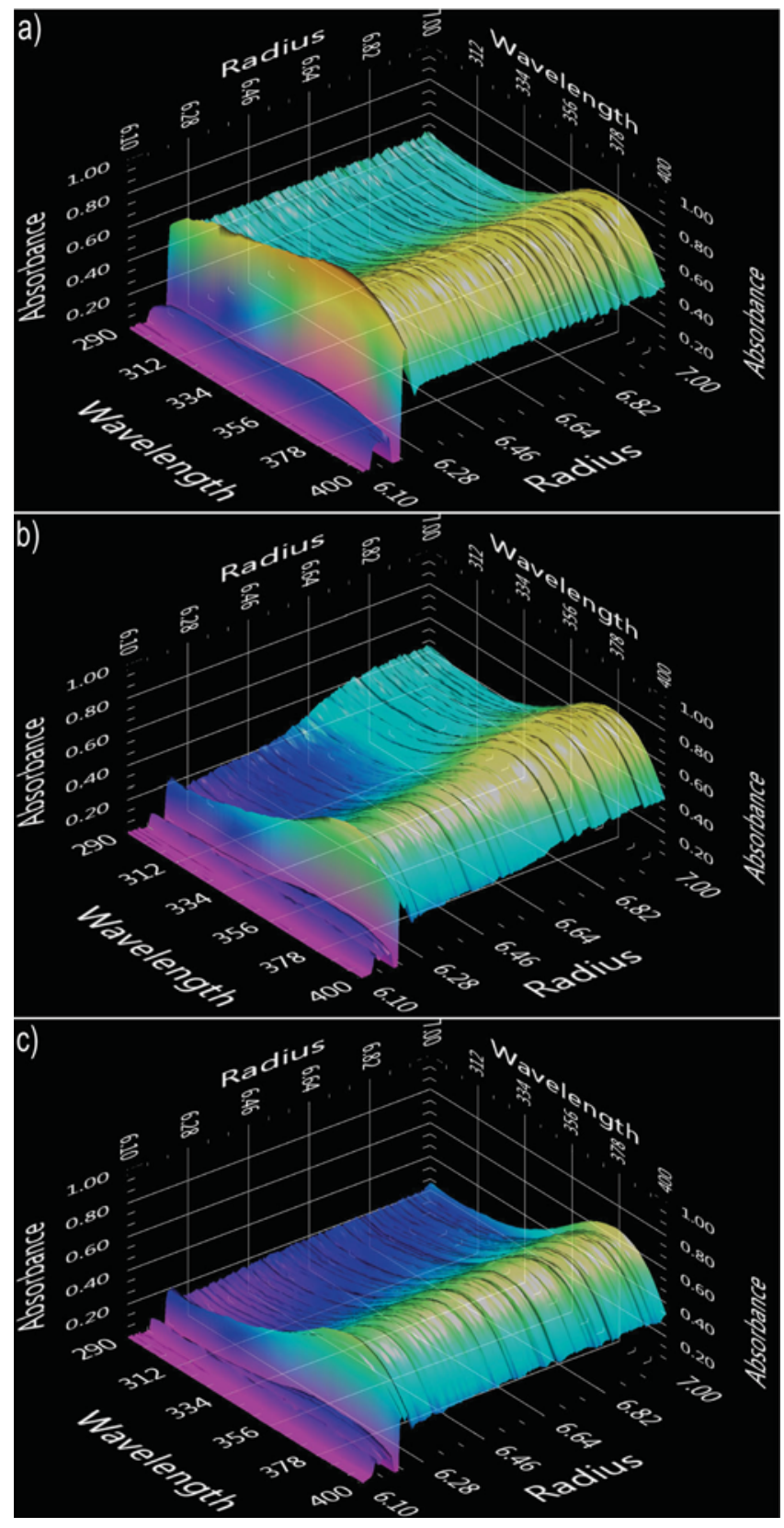

Figure 4. Three different scans (total of 100) of a multiwavelength analytical ultracentrifugation measurement of hybrid particles $\left(\mathrm{QDs}\left((\mathrm{HO})_{2}(\mathrm{O})\right.\right.$ $\left.\mathrm{P}^{-} \mathrm{C}_{6} \mathrm{H}_{4}-\mathrm{PF}\right)$ ) in solution at $10000 \mathrm{RPM}$ and $25^{\circ} \mathrm{C}$ : a) scan 1 ; b) scan 40 ; C) scan 100 .

the measurement cell (for complete data refer to Supporting Information). During centrifugation, however, a sedimentation shift to higher radii is observed for both QDs and part of the polyfluorene. After complete sedimentation of the QD-containing particles, no QD absorption is detected in the measurement cell and also polyfluorene absorption is reduced significantly throughout the entire measurement cell. The simultaneous sedimentation of QDs and a significant part of the polyfluorene during centrifugation confirms the formation of hybrid nanoparticles with direct attachment of the polyfluorene to the QD surface. In the case of QDs synthesized 
using oleyl amine, dodecylphosphonic acid and amino-functionalized polyfluorene as ligands $\left(\mathrm{QDs}\left(\mathrm{H}_{2} \mathrm{~N}-\mathrm{C}_{6} \mathrm{H}_{4}-\mathrm{PF}\right)\right)$, we estimate that $5 \%$ (SI) of the polyfluorene applied is attached to the QD surface under the conditions of AUC (highly diluted). By contrast, in case of the hybrid particles obtained by using only oleyl amine and phosphonic acid functionalized polyfluorene as ligands in the particle synthesis $\left(\mathrm{QDs}\left((\mathrm{HO})_{2}(\mathrm{O})\right.\right.$ P- $\mathrm{C}_{6} \mathrm{H}_{4}-\mathrm{PF}$ )), $45 \%$ of the polyfluorene applied is attached to the QDs. The observation of a much higher degree of functionalization in case of the $\mathrm{QDs}\left((\mathrm{HO})_{2}(\mathrm{O}) \mathbf{P}-\mathrm{C}_{6} \mathrm{H}_{4}-\mathrm{PF}\right)$ hybrid particles is consistent with the fact that there are less competing ligands present during the synthesis and the more strongly binding character of the phosphonic acid functionality used as an end-group.

We emphasize that strongly diluted solutions were used for AUC measurements to achieve manageable absorption depth of the solution, in agreement with the validity of Lambert-Beer analysis. The amount of bound polyfluorene is likely underestimated by the AUC measurement as any dissociation of bound polyfluorene from hybrid particles will be favored by high dilution, this appears particularly relevant for the more weakly coordinatively bound amino-functionalized polyfluorene. ${ }^{[31]}$ In addition to the above ensemble measurements, the optical quality of single hybrid nanoparticles was studied by micro photoluminescence spectroscopy. To ensure the observation of single particles, a highly diluted solution of hybrid particles $\left(\mathrm{QDs}\left(\mathrm{H}_{2} \mathbf{N}-\mathrm{C}_{6} \mathrm{H}_{4}-\mathrm{PF}\right)\right)$ in toluene was spin coated onto a fused quartz substrate. This procedure enables a spatial separation of QDs with attached amino-functionalized polyfluorene ligands from the remaining film of nonbound polyfluorene ligands. Illumination of a wide area on the sample surface with a continuous wave diode laser at a wavelength of $405 \mathrm{~nm}$ ensures the excitation of the hybrid particles and of non-bound polyfluorene ligands. Depending on whether the sample surface is covered only with polyfluorene or a single QD, different luminescence signatures were observed. Figure $\mathbf{5}$ depicts a typical image of the sample surface spectrally and spatially resolved via an imaging spectrometer and an electron-multiplying CCD camera. Narrow-band emission of a single QD is observed at $575 \mathrm{~nm}$, in contrast to broadband emission of the remaining polyfluorene ligands with a maximum at $490 \mathrm{~nm}$. The measurement reveals strong quenching of the polyfluorene emission in the surrounding of the QD, alluding to efficient energy transfer from the polyfluorene to the QD.

By comparing the spectra at the position of the QD and of the surrounding polyfluorene ligand film (Figure 5), we deduce a quenching efficiency on the order of $7 \%$. From the ratio of the areas of the emitting regions, we estimate that polyfluorene emission is completely $(100 \%)$ quenched in a radius of approximately $120 \mathrm{~nm}$ around the QD center. This clearly points out that the attachment of polyfluorene ligands to a colloidal QD does not lead to additional background emission by the former, rendering this method an ideal candidate for the synthesis of charged QDs for quantum optical applications.

Furthermore, we observed that sufficient increase of the pumping power leads not only to an increase of QD emission but is also accompanied by a fast photochemical bleaching of non-bound polyfluorene ligands. Finally, Figure 6 depicts the a)
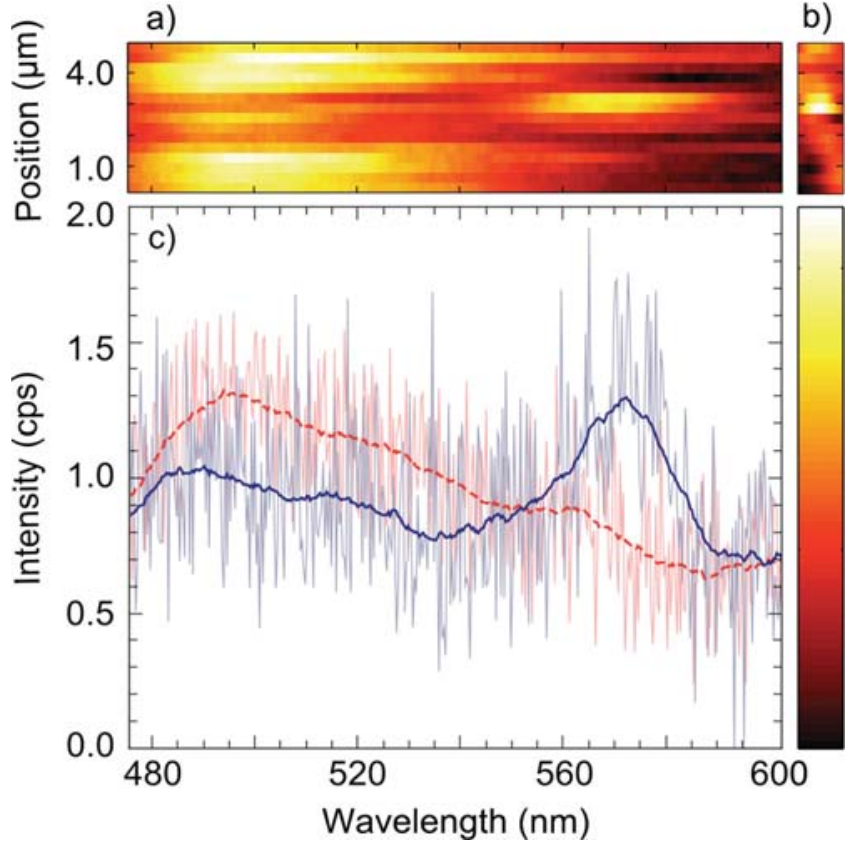

Figure 5. (a) Spatially resolved emission spectra of the polyfluorene and single QD (QDs $\left(\mathrm{H}_{2} \mathrm{~N}-\mathrm{C}_{6} \mathrm{H}_{4}-\mathrm{PF}\right)$ ), recorded in the micro photoluminescence setup. Spectral information is smoothened via an averaging window comprising 100 data points (approx. $32 \mathrm{~nm}$ ). (b) Signal intensity averaged over the entrance slit of the imaging spectrometer (1:1 aspect ratio), showing photoluminescence emission from the single QD. (c) Emission spectra of the QD (thin blue line, at a position of $3 \mu \mathrm{m}$ ) and of the surrounding polyfluorene film (thin red line, at a position of $4 \mu \mathrm{m}$ ). Thick lines of same color coding correspond to integration over $120 \mathrm{~s}$ spectrally smoothened with a moving average of 50 points.

time evolution of the QD spectrum exhibiting characteristic blinking behavior of a single QD $\left(\mathrm{QDs}\left(\mathrm{H}_{2} \mathrm{~N}-\mathrm{C}_{6} \mathrm{H}_{4}-\mathrm{PF}\right)\right)$. The bright emission from a single QD with a FWHM of $25 \mathrm{~nm}$ at room temperature does not only indicate a high quality of the nanoparticle but also directly proves the small level of inhomogeneous broadening when compared with the ensemble measurements in Figure 2 and in Figure 3. The on/off ratio in blinking dynamics is also favorable and yet another indication of the high quality of the nanoparticles prepared.
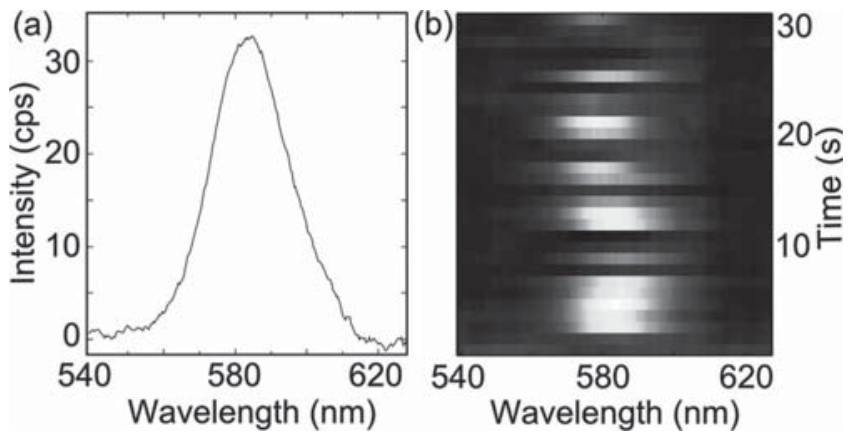

Figure 6. (a) Spectrum of the QD emission (QDs $\left(\mathrm{H}_{2} \mathrm{~N}-\mathrm{C}_{6} \mathrm{H}_{4}-\mathrm{PF}\right)$ ) recorded after bleaching of the polyfluorene emission. The full width at half maximum amounts to $25 \mathrm{~nm}$. (b) Evolution of the QD spectrum in time. The blinking behavior clearly indicates the presence of a single QD. All spectra are smoothened with a moving average of 100 points. 


\section{Conclusion}

Hybrid particles composed of an inorganic semiconductor nanocrystal with a shell of organic semiconductor ligands can be accessed directly by high temperature synthesis. Required functionalized fluorenes with amine or phosphonic acid end-groups suited for binding to the inorganic interface are accessible with high precision by a controlled Suzuki-Miyaura coupling polymerization with novel three-coordinated Pd(II) complexes as initiators. High optical quality quantum dot hybrids with narrow emission bands and bright emission are obtained. This is remarkable, considering that the established syntheses of quantum dots are highly optimized in an empirical fashion and are known to be very sensitive to small variations. At the same time, the hybrid particle dispersion possesses a high degree of colloidal stability. Analytical ultracentrifugation provides a rare quantitative insight into the extent of binding of the organic ligands to the nanocrystals in solution. For phosphonic acid functionalized polyfluorene, a substantial part (ca. half) of the ligand introduced in the nanocrystal synthesis indeed is bound tightly to the inorganic core. This efficient binding vs. aminofunctionalized polyfluorene (ca. $5 \%$ bound) can be related to a covalent nature of the phosphonic acid-CdSe interaction vs. a coordinative binding of amine. Single particle photoluminescence reveals an efficient energy transfer from the polyfluorene layer to the quantum dot and underlines low inhomogeneous broadening of the optical emission. Further studies aiming at a single electron transfer from the bound polyfluorene ligand to the inorganic nanoparticle are in progress.

\section{Supporting Information}

Supporting Information is available from the Wiley Online Library or from the author.

\section{Acknowledgements}

Financial support by the DFG (SFB767) is gratefully acknowledged. The authors thank Andreas Marquardt for MALDI-TOF measurements and Lars Bolk for GPC measurements.

[1] V. L. Colvin, M. C. Schlamp, A. P. Alivisatos, Nature 1994, 370, 354.

[2] J. Lee, V. C. Sundar, J. R. Heine, M. G. Bawendi, K. F. Jensen, Adv. Mat. 2000, 12, 1102.

[3] B. S. Mashford, M. Stevenson, Z. Popovic, C. Hamilton, Z. Zhou, C. Breen, J. Steckel, V. Bulovic, M. Bawendi, S. Coe-Sullivan, P. T. Kazlas, Nat. Photonics 2013, 7, 407.

[4] B. O'Regan, M. Grätzel, Nature 1991, 353, 737.
[5] N. C. Greenham, X. Peng, A. P. Alivisatos, Phys. Rev. B 1996, 54, 17628.

[6] W. U. Huynh, J. J. Dittmer, A. P. Alivisatos, Science 2002, 295, 2425.

[7] Z. Chen, H. Zhang, X. Du, X. Cheng, X. Chen, Y. Jiang, B. Yang, Energy Environ. Sci. 2013, 6, 1597.

[8] L. H. Zhao, Z. L. Zhou, Z. S. Guo, G. Gibson, J. A. Brug, S. Lam, J. Pei, S. S. Mao, J. Mater. Res. 2012, 27, 639.

[9] Y.-H. Chan, F. Ye, M. E. Gallina, X. Zhang, Y. Jin, I. C. Wu, D. T. Chiu, J. Am. Chem. Soc. 2012, 134, 7309.

[10] C. Negele, J. Haase, A. Budweg, A. Leitenstorfer, S. Mecking, Macromol. Rapid Commun. 2013, 34, 1145.

[11] C. Negele, J. Haase, A. Leitenstorfer, S. Mecking, ACS Macro Lett. 2012, 1, 1343.

[12] J. Merlein, M. Kahl, A. Zuschlag, A. Sell, A. Halm, J. Boneberg, P. Leiderer, A. Leitenstorfer, R. Bratschitsch, Nat. Photonics 2008, 2, 230.

[13] F. Sotier, T. Thomay, T. Hanke, J. Korger, S. Mahapatra, A. Frey, K. Brunner, R. Bratschitsch, A. Leitenstorfer, Nat. Phys. 2009, 5, 352.

[14] A. V. Akimov, A. Mukherjee, C. L. Yu, D. E. Chang, A. S. Zibrov, P. R. Hemmer, H. Park, M. D. Lukin, Nature 2007, 450, 402.

[15] A. G. Curto, G. Volpe, T. H. Taminiau, M. P. Kreuzer, R. Quidant, N. F. van Hulst, Science 2010, 329, 930.

[16] C. Javaux, B. Mahler, B. Dubertret, A. Shabaev, A. V. Rodina, A. L. Efros, D. R. Yakovlev, F. Liu, M. Bayer, G. Camps, L. Biadala, S. Buil, X. Quelin, J.-P. Hermier, Nat. Nanotechnol. 2013, 8, 206.

[17] B. C. Sih, M. O. Wolf, J. Phys. Chem. C 2007, 111, 17184.

[18] J. Locklin, D. Patton, S. Deng, A. Baba, M. Millan, R. C. Advincula, Chem. Mater. 2004, 16, 5187.

[19] Z.-S. Guo, L. Zhao, J. Pei, Z.-L Zhou, G. Gibson, J. Brug, S. Lam, S. S. Mao, Macromolecules 2010, 43, 1860.

[20] H. Skaff, K. Sill, T. Emrick, J. Am. Chem. Soc. 2004, 126, 11322.

[21] J. Xu, J. Wang, M. Mitchell, P. Mukherjee, M. Jeffries-El, J. W. Petrich, Z. Lin, J. Am. Chem. Soc. 2007, 129, 12828.

[22] A. Yokoyama, H. Suzuki, Y. Kubota, K. Ohuchi, H. Higashimura, T. Yokozawa, J. Am. Chem. Soc. 2007, 129, 7236.

[23] E. Elmalem, A. Kiriy, W. T. S. Huck, Macromolecules 2011, 44, 9057.

[24] Y. Nanashima, R. Shibata, R. Miyakoshi, A. Yokoyama, T. Yokozawa, J. Polym. Sci., Part A: Polym. Chem. 2012, 50, 3628.

[25] E. Elmalem, F. Biedermann, K. Johnson, R. H. Friend, W. T. S. Huck, J. Am. Chem. Soc. 2012, 134, 17769.

[26] C. S. Fischer, M .C. Baier, S. Mecking, J. Am. Chem. Soc. 2013, 135, 1148.

[27] C. B. Murray, D. J. Norris, M. G. Bawendi, J. Am. Chem. Soc. 1993 $115,8706$.

[28] M. B. Mohamed, D. Tonti, A. Al-Salman, A. Chemseddine, M. Chergui, J. Phys. Chem. B 2005, 109, 10533.

[29] B. Mahler, P. Spinicelli, S. Buil, X. Quelin, J.-P. Hermier, B. Dubertret, Nat. Mater. 2008, 7, 659

[30] R. Gomes, A. Hassinen, A. Szczygiel, Q. Zhao, A. Vantomme, J. C. Martins, Z. Hens, J. Phys. Chem. Lett. 2011, 2, 145.

[31] A. Hassinen, I. Moreels, C. de Mello Donegá, J. C. Martins, Z. Hens, J. Phys. Chem. Lett. 2010, 1, 2577.

[32] A. J. Morris-Cohen, M. D. Donakowski, K. E. Knowles, E. A. Weiss, J. Phys. Chem. C 2010, 114, 897.

[33] H. Strauss, E. Karabudak, S. Bhattacharyya, A. Kretzschmar, W. Wohlleben, H. Cölfen, Colloid Polym. Sci. 2008, 286, 121.

[34] S. K. Bhattacharyya, P. Maciejewska, L. Börger, M. Stadler, A. M. Gülsün, H. B. Cicek, H. Cölfen, Prog. Colloid Polym. Sci. 2006, 131, 9. 